\title{
Cortical Thickness and Subcortical Gray Matter Volume in Pediatric Anxiety Disorders
}

\author{
Andrea L Gold", , Elizabeth R Steuber', Lauren K White ${ }^{1,2}$, Jennifer Pacheco ${ }^{3}$, Jessica F Sachs', \\ David Pagliaccio', Erin Berman', Ellen Leibenluft' and Daniel S Pine' \\ 'Emotion and Development Branch, National Institute of Mental Health, National Institutes of Health, Bethesda, MD, USA; ${ }^{2}$ Department of Child \\ and Adolescent Psychiatry and Behavioral Sciences, Lifespan Brain Institute, Children's Hospital of Philadelphia, Philadelphia, PA, USA; ${ }^{3}$ Office of the \\ Director, National Institute of Mental Health, National Institutes of Health, Bethesda, MD, USA
}

\begin{abstract}
Perturbations in the prefrontal cortex (PFC), hippocampus, and amygdala are implicated in the development of anxiety disorders. However, most structural neuroimaging studies of patients with anxiety disorders utilize adult samples, and the few studies in youths examine small samples, primarily with volume-based measures. This study tested the hypothesis that cortical thickness of PFC regions and gray matter volume of the hippocampus and amygdala differ between pediatric anxiety disorder patients and healthy volunteers (HVs). High-resolution 3-Tesla TI-weighted MRI scans were acquired in I5I youths (75 anxious, 76 HV; ages 8-I8). Analyses tested associations of brain structure with anxiety diagnosis and severity across both groups, as well as response to cognitive-behavioral therapy in a subset of 53 patients. Cortical thickness was evaluated both within an a priori PFC mask (small-volume corrected) and using an exploratory whole-brain-corrected $(p<0.05)$ approach. Anxious relative to healthy youths exhibited thicker cortex in the left ventromedial PFC (vmPFC) and left precentral gyrus. Both anxiety diagnosis and symptom severity were associated with smaller right hippocampal volume. In patients, thinner cortex in parietal and occipital cortical regions was associated with worse treatment response. Pediatric anxiety was associated with structural differences in vmPFC and hippocampus, regions implicated in emotional processing and in developmental models of anxiety pathophysiology. Parietal and occipital cortical thickness were related to anxiety treatment response but not baseline anxiety.

Neuropsychopharmacology (2017) 42, 2423-2433; doi:I0.1038/npp.2017.83; published online 31 May 2017
\end{abstract}

\section{INTRODUCTION}

Neuroscience research implicates perturbed brain development in anxiety disorders, particularly in the prefrontal cortex (PFC), hippocampus, and amygdala (Brühl et al, 2014a; Etkin and Wager, 2007). However, most structural neuroimaging studies utilize adult samples; the few pediatric studies examine small samples, primarily with volume-based measures. Moreover, major questions exist regarding the relationship between brain structure and treatment response, but no prior study of anxious youths considers this issue. This study relates brain structure to pre-treatment anxiety and response to cognitive-behavioral therapy (CBT) in youths.

Cortical and subcortical regions, including the PFC, insula, amygdala, and hippocampus, are engaged during emotional processes that relate to anxiety symptoms such as threat learning and attention (Britton et al, 2011; White et al,

*Correspondence: Dr AL Gold, Emotion and Development Branch, National Institute of Mental Health, National Institutes of Health, Building I5K, MSC-2670, Bethesda, MD 20892-2670, USA, Tel: + I 30 I 34733400 17; Fax: + I 301 40220 10; E-mail: andrea.gold@nih.gov

Received I4 March 2017; revised II April 2017; accepted 17 April 2017; accepted article preview online 24 April 2017 in press). Prior studies link anxiety to structural alterations in these regions (Brühl et al, 2014a; Shang et al, 2014). A meta-analysis of anxiety disorders (Shang et al, 2014), including mostly adult studies, found both larger and smaller gray matter volume (GMV) in PFC sub-regions. Findings in the few pediatric studies are inconsistent: patients manifest larger and smaller GMV across various PFC sub-regions, with little consistency (for summary of previous findings see Supplementary Table S1; Liao et al, 2014; Milham et al, 2005; Mueller et al, 2013; Strawn et al, 2013, 2015). One study also found larger insula GMV (Mueller et al, 2013). Some studies relate smaller hippocampal GMV to pediatric anxiety disorders (Mueller et al, 2013) or symptoms (Koolschijn et al, 2013) while several show no differences (De Bellis et al, 2000; Liao et al, 2014; Milham et al, 2005; Strawn et al, 2013). GMV findings for the amygdala, such as for the PFC, show larger (De Bellis et al, 2000; Jones et al, 2015), and smaller (Milham et al, 2005; Mueller et al, 2013; Strawn et al, 2015) volumes in anxious relative to healthy youths, while other studies find no group differences (Liao et al, 2014; Strawn et al, 2013). However, estimates in these studies are imprecise, as no study included over 40 patients. The current study compares brain structure in 75 pediatric anxiety patients and 76 healthy volunteers (HVs). 
While multiple investigations relate anxiety to altered cortical structure, most studies rely on volume-based methods (Supplementary Table S1). This approach has limitations, as volumetric findings can reflect differences in thickness, surface area, and/or folding. Thickness and surface area show unique developmental trajectories, may be genetically distinct, and may relate differentially to volume (Panizzon et al, 2009; Raznahan et al, 2011; Wierenga et al, 2014; Winkler et al, 2010). This may explain why different between-subject patterns arise for measures of cortical thickness, surface area, and volumes (Hutton et al, 2009; Supplementary Table S1). While a growing number of studies consider surface-based measures, in adult anxiety (Brühl et al, 2014b; Frick et al, 2013; Syal et al, 2012), only one prior study, which included only 13 patients, examines cortical thickness in pediatric anxiety (Strawn et al, 2014).

Identifying perturbations in brain structure also might inform research on therapeutics. Studies have begun to use structural neuroimaging to predict treatment response, although the few studies to attempt to do so in anxiety include only adults (Bryant et al, 2008; Fullana et al, 2014; Hoexter et al, 2012). Such research may improve treatment selection for pediatric patients, many of whom require additional treatment following CBT, the best-established behavioral treatment (Mohatt et al, 2014). CBT aims to reduce symptoms via behavioral (eg, exposure) and cognitive strategies (eg, reappraisal). Certain brain regions underlying the processes recruited during CBT may be particularly informative of treatment response. For instance, exposure relates to extinction learning and recall, processes supported by the amygdala, hippocampus, and ventromedial PFC (vmPFC) (Milad and Quirk, 2012). Cognitive reappraisal of emotion involves the amygdala and cognitive control regions including lateral PFC (Buhle et al, 2014). The three neuroimaging studies to date that examined treatment response in anxious youths implicated amygdala and PFC function, and amygdala-PFC and amygdala-insula functional connectivity (Kujawa et al, 2016; McClure et al, 2007; White et al, in press). We are unaware of any structural neuroimaging studies examining treatment response in pediatric anxiety.

This study used automated measures of cortical thickness and subcortical volume to address two goals. First, this study tested the hypothesis that brain structure relates to pretreatment anxiety. Prior studies support the prediction that anxious relative to non-anxious youths would show thicker cortex in the PFC, particularly vmPFC, and insula, and smaller hippocampal and amygdala GMV, although other studies suggest larger amygdala GMV (Supplementary Table S1). Second, exploratory analyses tested whether brain structure predicts treatment response in a subset of 53 patients completing CBT, 40 of whom concurrently received attention bias modification therapy (ABMT). Clinical outcomes and functional neuroimaging in an overlapping sample are reported in White et al, in press. Initial fMRI findings highlight similar regions relating to treatment response (Kujawa et al, 2016; McClure et al, 2007; White et al, in press); however, our hypotheses about structure were non-directional as no prior pediatric studies examine this association.

\section{MATERIALS AND METHODS}

\section{Participants}

Participants included 151 children and adolescents (75 anxious, $76 \mathrm{HV}$ ) ages $8-18$ years (mean $=12.47$ years, $\mathrm{SD}=2.78$ years; 87 female) recruited from the community to participate in research at the National Institute of Mental Health (NIMH; see Supplementary Information for details). Exclusion criteria included IQ $<70$, current psychotropic medications, MRI contraindications, or physical health problems.

Parents and 18-year-olds provided written informed consent and minors provided written assent in accordance with the NIMH Institutional Review Board. Voxel-based morphometry (VBM) analyses from a subset of 56 participants (30 healthy, 26 anxious) were previously reported in a multi-group study (Gold et al, 2016); additionally, VBM analyses of MRI data obtained at earlier time points from 15 participants (6 healthy, 9 anxious) were previously reported (Adleman et al, 2012; Gold et al, 2016; Mueller et al, 2013). However, no FreeSurfer-based analyses have been reported from any participants.

\section{Anxiety Diagnosis and Severity Measures}

Axis I diagnoses were determined via the Kiddie Schedule for Affective Disorders and Schizophrenia for School-Age Children-Present and Lifetime Version (KSADS-PL) (Kaufman et al, 1997) by clinicians trained to acceptable levels of reliability (kappa $>0.80$ ) and confirmed by senior psychiatrists during independent assessments. HVs were diagnosis free. Patients met DSM-5 criteria for a primary diagnosis of anxiety (generalized, separation, and/or social anxiety disorder) that required treatment.

In addition to the primary, dichotomous measure of anxiety diagnosis, a dimensional measure of anxiety symptom severity was obtained using the Screen for Child Anxiety-Related Emotional Disorders (SCARED) (Birmaher et al, 1997). The SCARED assesses anxiety symptoms over the past 3 months and was collected within 2 months before or after scanning. SCARED data were available for 108 participants (37 healthy, 71 anxious). Anxiety severity was based on the average of total anxiety scores from the parent and child SCARED ratings, which were correlated $(r=0.54$, $p<0.001)$. Dimensional analyses tested associations with brain structure across the full sample. Examining associations within the patients only restricts the range of scores ignoring cases at the low end of the scale. However, these results are reported for analyses showing significant associations across the full sample, for thoroughness. Exploratory analyses tested the generalized and social anxiety subscales separately, as well as specificity among generalized $v s$ social anxiety diagnoses (Supplementary Information).

\section{Anxiety Treatment Measures}

Patients underwent CBT with trained psychologists based on the Coping Cat protocol (Kendall and Hedtke, 2006); (Supplementary Information). Clinician-based symptom ratings were obtained at baseline, mid-treatment, and post treatment using the 50-item Pediatric Anxiety Rating Scale (PARS) (RUPP Anxiety Study Group, 2002). Clinician raters 
initially were trained to acceptable levels of reliability (kappa $>0.80)$ on all interview assessments. Secondary analyses relating brain structure to treatment measures examined 53 patients who met three criteria. First, patients included in treatment analyses attended three or more CBT sessions and remained medication-free ( $n=7$ excluded). Second, patients were required to have PARS data at baseline and post treatment ( $n=9$ excluded). Finally, MRI data were required to be available within 6 weeks before or 3 weeks after baseline clinician ratings were collected ( $n=6$ excluded; See Supplementary Information for details).

The primary treatment response measure was posttreatment PARS ratings, reflecting the gold standard continuous treatment measure assessed in prior studies (RUPP Anxiety Study Group, 2001). PARS analyses controlled for baseline anxiety. Finally, $n=40$ of the 53 patients received placebo $(n=23)$ or active $(n=17)$ forms of ABMT concurrent with CBT. Clinical outcomes of the ABMT trial and associations of functional neuroimaging measures with ABMT and CBT are reported elsewhere (White et al, in press). Moderation analyses examining ABMT-specific treatment response are limited by small sample size, but reported in the Supplementary Information for thoroughness.

\section{MRI Acquisition and Processing}

High-resolution structural MRI images $(1 \times 1 \times 1 \mathrm{~mm})$ were acquired on a 3-Tesla MR750 GE scanner with a 32-channel head coil; see Supplementary Methods for acquisition parameters. Processing utilized standard procedures in the FreeSurfer image analysis software suite (Version 5.3, http:// surfer.nmr.mgh.harvard.edu), including cortical surface reconstruction, cortical thickness estimation, cortical volume parcellation, and subcortical volume segmentation. For details, see Supplementary Methods. Prior to analysis, all MRI scans were run through the FreeSurfer reconstruction process and were visually inspected for image artifacts, leading to exclusion of 21 participants (17 anxious, 4 healthy). Seventy-six HVs were selected from a larger pool of $n=83$ to create a comparison sample group matched with the 75 anxiety patients. This resulted in a final sample $(N=151)$ in which the groups did not differ in age, sex, IQ, or socioeconomic status, all $p s>0.1$ (Table 1 ).

\section{Statistical Analysis}

Associations of brain structure with anxiety diagnosis $(N=151)$, severity $(N=108)$, and treatment response $(N=53)$ were tested. Brain structure was assessed using two measures: cortical thickness and subcortical GMV. To account for potential associations of age and sex with brain structure, diagnosis-by-age and diagnosis-by-sex interactions were tested in separate models. There were no significant age-by-diagnosis or sex-by-diagnosis interactions for cortical thickness (whole-brain-corrected) or subcortical GMV (all $p s>0.26)$. Given that the age- and sex-by-diagnosis interactions were non-significant, and that there were no group differences in age or sex, neither variable nor their interactions with diagnosis were included in any of the subsequent hypothesis testing models.

All cortical thickness analyses were conducted separately for each hemisphere with the FreeSurfer QDEC surface- based group analysis tool, Bonferroni-corrected for laterality (ie, two measurements), using a $10 \mathrm{~mm}$ smoothing kernel and were based on two approaches to multiple comparison corrections. First, we conducted exploratory whole-braincorrected vertex-wise analyses, with vertex- and cluster-wise significance set to $p<0.05$ based on Monte Carlo simulations via QDEC. Second, we tested a priori hypotheses in PFC and insular cortex, structures implicated in prior studies (Mueller et al, 2013; Strawn et al, 2013, 2014, 2015). On the basis of their connections and topology, agranular insular cortex represents an integral part of the orbital neocortex (Murray et al, 2017). Thus, both insular and PFC regions were included in the a priori mask (see Supplementary Figure S1 for details), referred to as the 'PFC mask' or 'PFC-corrected threshold' for simplicity. Using vertex-wise $p<0.05$, the PFC-wide cluster-wise significance was set to $p<0.05$ based on Monte Carlo simulations. Supplementary Materials report associations of anxiety diagnosis and severity with cortical thickness for the insula and PFC sub-regions, along with bootstrapped confidence intervals (Supplementary Tables S4-S5). This region-of-interest approach was not intended for hypothesis testing, but rather to inform future research by showing the magnitude and direction of differences in regions failing to pass correction for multiple comparisons.

Finally, subcortical GMV analyses were conducted in two a priori regions-of-interest generated by FreeSurfer automated segmentation: hippocampus and amygdala. Subcortical GMV was tested separately for each hemisphere, with alpha $=0.025$ following Bonferroni correction for laterality. Multiple regression analyses tested associations of subcortical GMV with the three clinical measures, separately, in each region. All subcortical GMV analyses controlled for estimated total intracranial volume (ICV).

\section{RESULTS}

For sample characteristics by group, including mean thickness and ICV, see Table 1 .

\section{Anxiety Diagnosis}

Cortical thickness. One cluster in the left precentral gyrus survived whole-brain correction, with thicker cortex in anxious relative to HV participants (Peak Talairach Coordinates (XYZ): $-25.4,-9.3,46.3 ; 1023.81 \mathrm{~mm}^{2} ; 2408$ vertices; Cohen's $d=0.58$; Figure 1a). A left vmPFC cluster survived the PFC-corrected threshold, with thicker cortex in anxious than HV participants (peak: -7.2, 27.4, - 15.5; $612.25 \mathrm{~mm}^{2}$; 1128 vertices; Cohen's $d=0.52$; Figure $1 \mathrm{~b}$ ). No other clusters survived either threshold.

Subcortical GMV. Anxiety diagnosis predicted smaller GMV in the right hippocampus, but not the left hippocampus nor the amygdala (Table 2).

\section{Anxiety Severity}

Cortical thickness. No significant clusters emerged using either the whole-brain-corrected or PFC-corrected thresholds for the anxiety severity analyses. 
Brain morphometry in pediatric anxiety

AL Gold et al

Table I Sample Characteristics by Anxiety Diagnosis

\begin{tabular}{|c|c|c|c|c|}
\hline Characteristic $^{\mathbf{a}}$ & $\begin{array}{c}\text { Anxious }(N=75) \\
\text { Mean (SD) }\end{array}$ & $\begin{array}{l}\text { Healthy }(N=76) \\
\text { Mean }(\mathrm{SD})\end{array}$ & $t$ & $p$-value \\
\hline Age range (years) & $8.09-18.08$ & $8.09-17.82$ & & \\
\hline IQ (WASI) ${ }^{\mathrm{b}}$ & $115.80(15.15)$ & $112.89(11.19)$ & 1.34 & 0.18 \\
\hline SES & $31.15(14.46)$ & $34.95(14.65)$ & -1.53 & 0.13 \\
\hline SCARED Total-Child & $27.73(|1.7|)$ & $9.60(9.74)$ & 8.07 & $<0.001$ \\
\hline SCARED-SoPH Child & $7.65(4.35)$ & $3.14(3.56)$ & 5.43 & $<0.001$ \\
\hline SCARED-SoPH Parent & $8.45(4.25)$ & $1.65(2.20)$ & 10.97 & $<0.001$ \\
\hline SCARED-SoPH P/C Avg & $8.05(3.66)$ & $2.39(2.42)$ & 9.60 & $<0.00$ I \\
\hline SCARED-Gen Child & $8.76(4.76)$ & $2.54(2.66)$ & 8.71 & $<0.001$ \\
\hline SCARED-Gen Parent & | $1.07(4.03)$ & $1.03(1.66)$ & 18.23 & $<0.001$ \\
\hline PARS-Baseline ${ }^{c}$ & 17.11 (3.07) & - & - & - \\
\hline PARS-Post range ${ }^{c}$ & $1.00-22.00$ & - & - & - \\
\hline PARS-Post ${ }^{c}$ & $12.60(4.34)$ & - & - & - \\
\hline $\mathrm{RH}$ mean thickness $(\mathrm{mm})$ & $2.69(0.12)$ & $2.66(0.12)$ & 1.17 & 0.25 \\
\hline LH mean thickness $(\mathrm{mm})$ & $2.70(0.12)$ & $2.67(0.11)$ & 1.32 & 0.19 \\
\hline Intracranial volume $\left(\mathrm{mm}^{3}\right)$ & I,494,815.36 (169,509.69) & I,535,033.25 ( I 61, 104.22) & -1.50 & 0.14 \\
\hline Characteristic & $\begin{array}{c}\text { Anxious }(N=75) \\
N(\%)\end{array}$ & $\begin{array}{c}\text { Healthy }(N=76) \\
N(\%)\end{array}$ & $\chi^{2}$ & $p$-value \\
\hline Female & $44(58.67 \%)$ & $43(56.58 \%)$ & 0.07 & 0.80 \\
\hline ODD & | (I.30\%) & 0 & - & - \\
\hline PDD-NOS & 0 & 0 & - & - \\
\hline Tic/Tourette $d x$ & $3(4.00 \%)$ & 0 & - & - \\
\hline SM & $4(5.33 \%)$ & 0 & - & - \\
\hline Elimination $\mathrm{dx}$ & $4(5.33 \%)$ & 0 & - & - \\
\hline Prior psychotropic medications ${ }^{d}$ & $9(12.0 \%)$ & - & - & - \\
\hline Antidepressant/anxiolytic & $4(5.33 \%)$ & - & - & - \\
\hline Anti-ADHD/stimulant & $5(6.67 \%)$ & - & - & - \\
\hline
\end{tabular}

Abbreviations: ADHD, attention-deficithyperactivity disorder; ANX, anxious youths; Avg, average; C, child; CDI, child depression inventory; CGl, Clinical Global Impressions Scale Improvement; Dx, disorder; GAD, generalized anxiety disorder; Gen, generalized anxiety; HV, healthy youths; IQ, intelligence quotient; LH, left hemisphere; MDD, major depressive disorder; ODD, oppositional defiant disorder, P, parent; PARS, Pediatric Anxiety Rating Scale; PDD-NOS, pervasive developmental disorder not otherwise specified; RH, right hemisphere; SAD, separation anxiety disorder, SCARED, Screen for Child Anxiety-Related Emotional Disorders; SES, socioeconomic status; SM, selective mutism; SoPH, social anxiety disorder; STAI, Spielberger State-Trait Anxiety Inventory; WASI, Wechsler Abbreviated Scale of Intelligence.

aData unavailable: IQ (WASI): I HV, 0 ANX; SES: I I HV, 2 ANX; SCARED: 39 HV, 4 ANX; STAI-State: I 3 HV, 0 ANX; CDI: 33 HV, 2 ANX.

${ }^{b} \mathrm{Q}$ ascertained by the Vocabulary and Matrix Reasoning subscales of the WASI.

'PARS data reported for the subset of $n=53$ patients included in the treatment response analyses.

${ }^{\mathrm{d}}$ All patients were free of psychotropic medications at the time of scanning. 

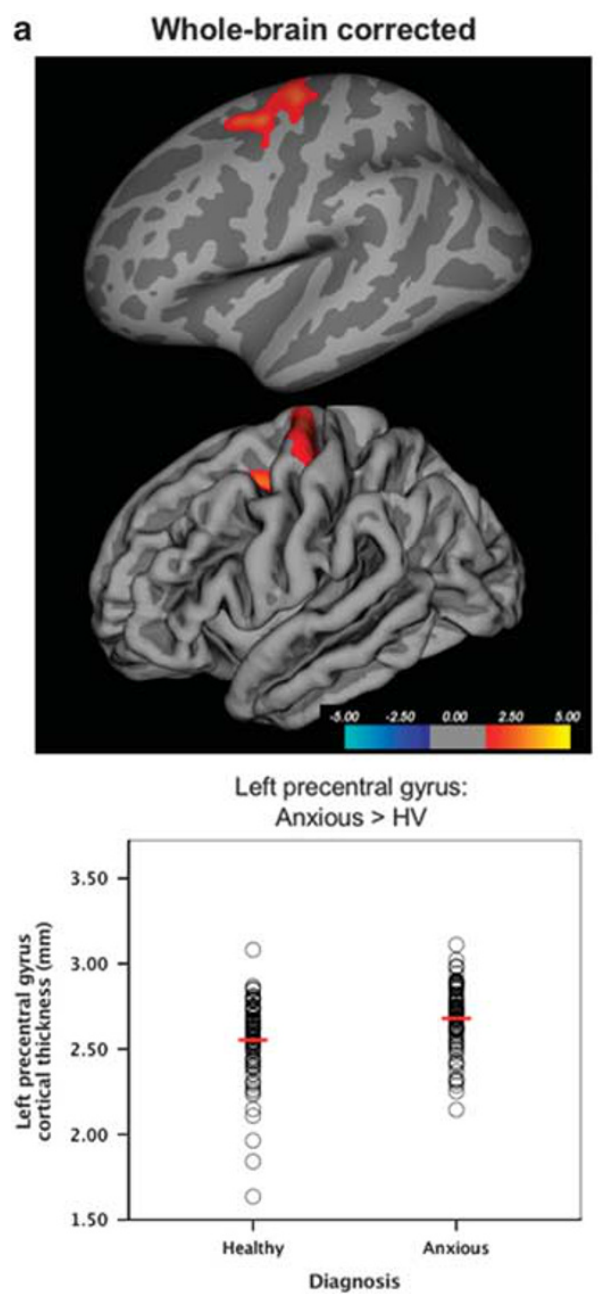

b

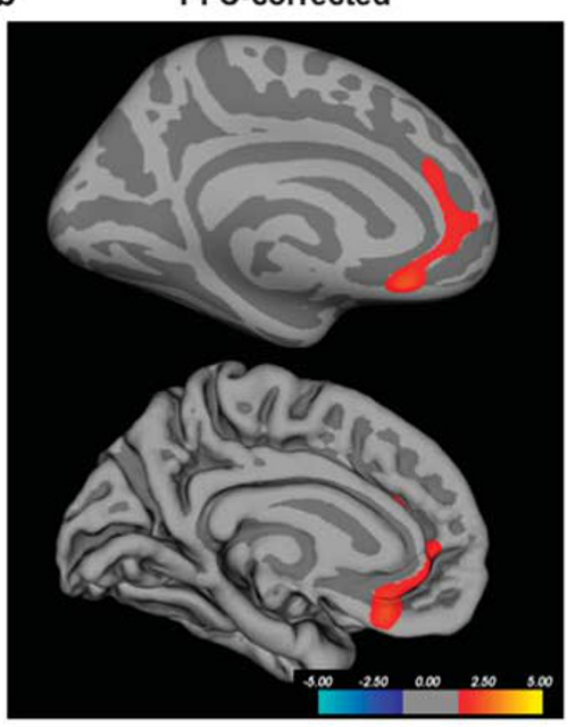

Left vmPFC:

Anxious $>\mathrm{HV}$

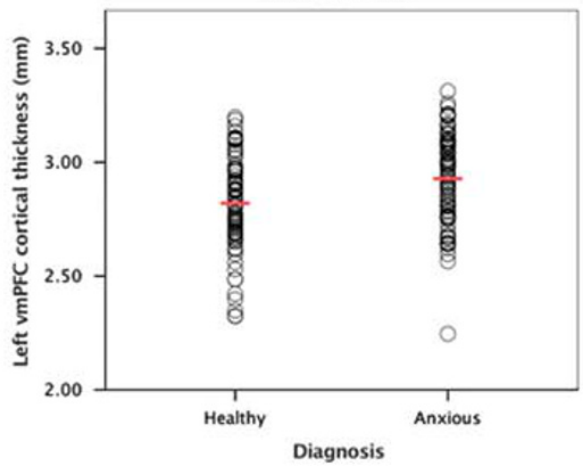

Figure I Cortical thickness in anxious relative to healthy youths. (a) On the basis of whole-brain vertex-wise correction ( $p<0.05$, corrected), greater cortical thickness in the anxious relative to healthy volunteer (HV) participants was observed in the left precentral gyrus. Results are shown on the inflated (top row) and pial (bottom row) surfaces. Mean thickness values for the left precentral gyrus cluster are plotted for individual participants in the healthy and anxious groups, separately, with group mean values indicated via horizontal red lines. (b) On the basis of the prefrontal cortex (PFC)-corrected threshold, greater cortical thickness was also observed in anxious relative to HV participants in the ventromedial prefrontal cortex (vmPFC), spanning medial orbitofrontal cortex and rostral anterior cingulate cortex. Results are shown on the inflated (top row) and pial (bottom row) surfaces. Mean thickness values for the left vmPFC cluster are plotted for individual participants in the healthy and anxious groups, separately, with group mean values indicated via horizontal red lines.

Subcortical GMV. Greater anxiety severity predicted smaller GMV in the right hippocampus, but neither the left nor right amygdala (Table 2/Figure 2). The association in the right hippocampus was non-significant when tested in the anxious group only.

\section{Anxiety Treatment Response}

Cortical thickness. Thinner cortex in five clusters in parietal and occipital cortices was associated with worse treatment response, ie, higher anxiety symptoms post treatment, controlling for baseline symptoms (Figure 3). The whole-brain-corrected analysis revealed two left hemisphere clusters: one in superior parietal cortex, spanning the lateral occipital cortex and cuneus (peak: - 23.8, - 65.7, 27.7; $2648.7 \mathrm{~mm}^{2} ; 4100$ vertices), and the other in inferior parietal cortex, extending to the supramarginal gyrus (peak: -42.9 , $-51.7,23.1 ; 1594.14 \mathrm{~mm}^{2} ; 3235$ vertices). In the right hemisphere, one cluster emerged in superior parietal cortex, extending to lateral occipital cortex (peak: 18.5, - 81.7, 23.2; $1534.40 \mathrm{~mm}^{2} ; 2448$ vertices) and two in lingual gyrus, one extending to fusiform gyrus (peak: 18.9, -73.6, -5.0; $1168.65 \mathrm{~mm}^{2} ; 1537$ vertices) and another extending to the pericalcarine cortex (peak: 22.2, $-47.1,-0.9 ; 1045.97 \mathrm{~mm}^{2}$; 1984 vertices). No clusters emerged for the PFC-corrected threshold.

Subcortical GMV. Treatment response was not associated with subcortical GMV in the hippocampus or amygdala (Supplementary Table S3).

\section{DISCUSSION}

This study generated three key findings. First, thicker cortex in the vmPFC was observed in anxious relative to healthy youths. Second, smaller hippocampal volume was associated with anxiety diagnosis and severity. Finally, in secondary analyses examining patients completing CBT, thinner 
Table 2 Associations of Anxiety Diagnosis and Severity with Subcortical Gray Matter Volumes ${ }^{a}$

Anxiety diagnosis group differences $(N=151)$

\begin{tabular}{|c|c|c|c|c|c|c|}
\hline Region & $\begin{array}{l}\text { Anxious }(N=75) \\
\text { Mean }(S D)\left(\mathrm{mm}^{3}\right)\end{array}$ & $\begin{array}{l}\text { Healthy }(N=76) \\
\text { Mean }(S D)\left(\mathrm{mm}^{3}\right)\end{array}$ & $\beta$ & Cohen's $d^{b}$ & $t$ & $p$-value \\
\hline \multicolumn{7}{|c|}{ Hippocampus } \\
\hline Right & $4005.04(465.36)$ & $4215.23(453.56)$ & -0.32 & 0.38 & -2.36 & 0.02 \\
\hline Right & | $491.30(177.53)$ & | 527.67 (|96.92) & -0.04 & 0.06 & -0.34 & 0.73 \\
\hline
\end{tabular}

Continuous associations with anxiety severity ${ }^{c}(N=108)$

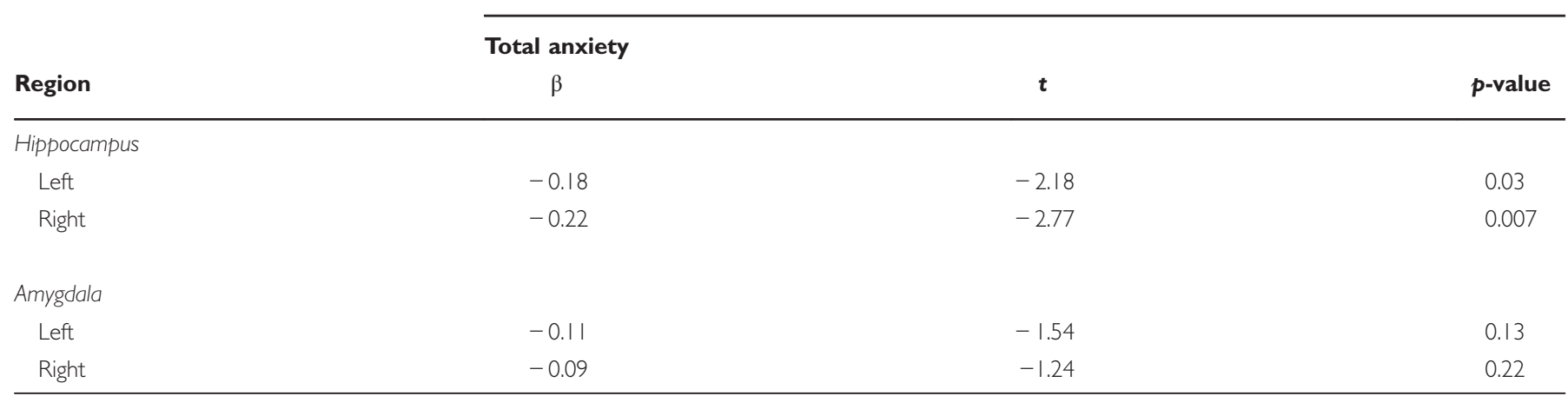

Abbreviations: HV, healthy volunteer, SCARED, Screen for Child Anxiety-Related Emotional Disorders.

${ }^{a}$ Analyses control for estimated total intracranial volume (ICV) to examine regional GMV (independent of total brain size).

${ }^{b}$ Cohen's $d$ calculated on the marginal means, controlling for ICV.

'Anxiety severity scores measured by the SCARED (Birmaher et al, 1997) and based on the average of child and parent reports within 60 days before or after the scan; data available for 108 participants (37 HV, $7 \mathrm{I}$ anxious).

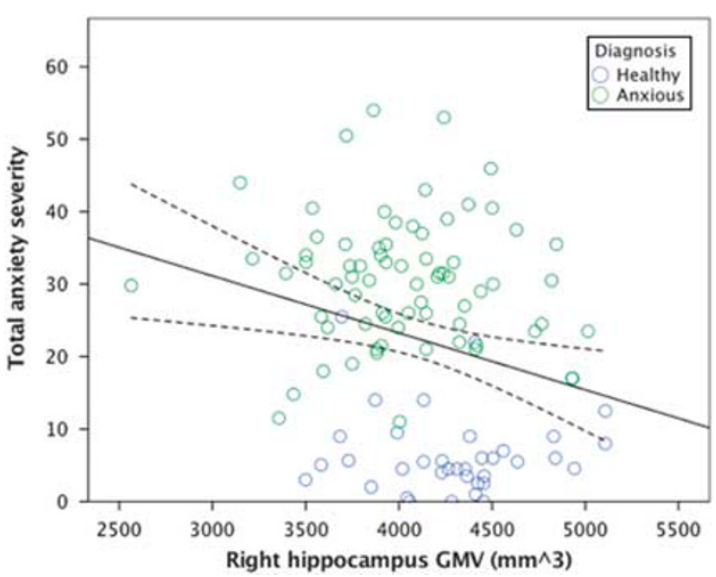

Figure 2 Smaller hippocampal volumes associated with anxiety severity. Across all participants with available data $(N=108)$, total anxiety severity was inversely related to gray matter volume (GMV) in the right hippocampus $(\beta=-0.22)$.

occipital and parietal cortex was associated with worse treatment response.

In anxious relative to healthy youths, thicker cortex was observed in the vmPFC, including ventral ACC, with a medium effect size. This region shares neuroanatomical connections with the amygdala, hippocampus, and lateral PFC, and thus is a component of corticolimbic circuitry linked to perturbations in anxiety pathology (Brühl et al, 2014a; Price and Drevets, 2010). The vmPFC is consistently implicated in neuroimaging studies of pathological anxiety and emotional processes, such as fear extinction recall and fear generalization (Britton et al, 2013; Cha et al, 2014; Milad et al, 2005; Milad and Quirk, 2012). Of note, the current findings replicate the only prior pediatric anxiety cortical thickness study, in which Strawn et al (2014) found similarly thicker cortex in ventromedial and ventrolateral PFC in adolescents with generalized anxiety disorder. In the current study, anxiety diagnosis also related to thicker cortex in the left precentral gyrus. This finding appears less consistent with the prior literature, as Strawn et al (2014) found no differences in precentral gyrus and only one volumetric study (Strawn et al, 2013) observed larger GMV. Furthermore, no regions showed thinner cortex associated with anxiety diagnosis or symptoms; this is consistent with Strawn et al (2014) and the largest adult anxiety thickness study (Brühl et al, 2014b). However, other studies report thinner cortex of various regions in adult anxiety disorders such as social anxiety disorder (Frick et al, 2013; Syal et al, 2012). Thus, available data in youths find only thicker cortex in PFC, and more research comparing adult and pediatric anxiety in the same study is needed. Moreover, the significance of thicker cortex in patients is still debated. Basic science in nonhuman animal studies is needed to tease apart mechanisms 
a

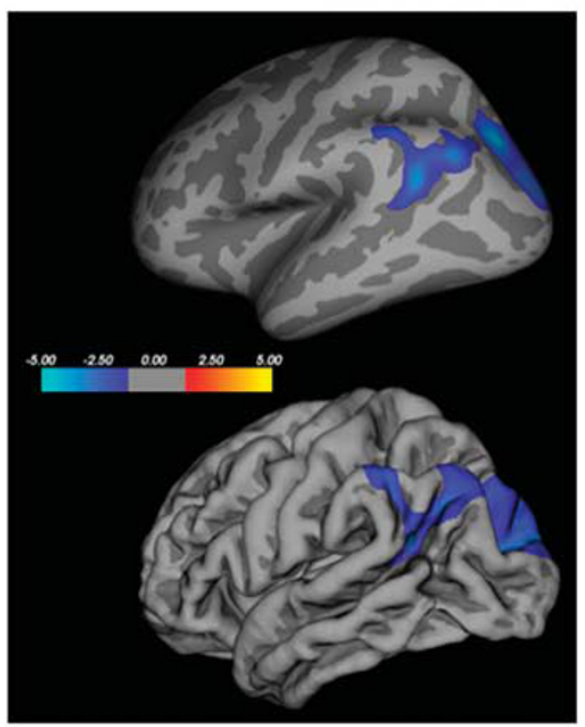

Whole-brain corrected: Left hemisphere
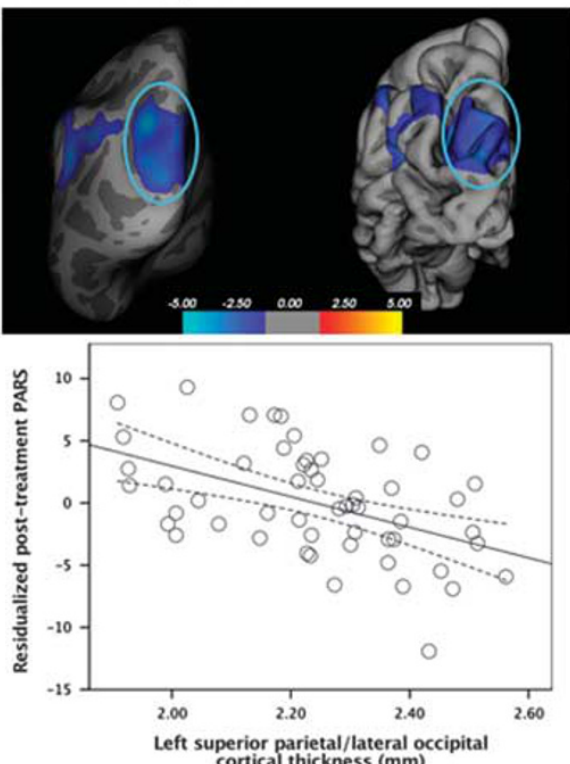

cortical thickness (mm)

b Whole-brain corrected, Right hemisphere
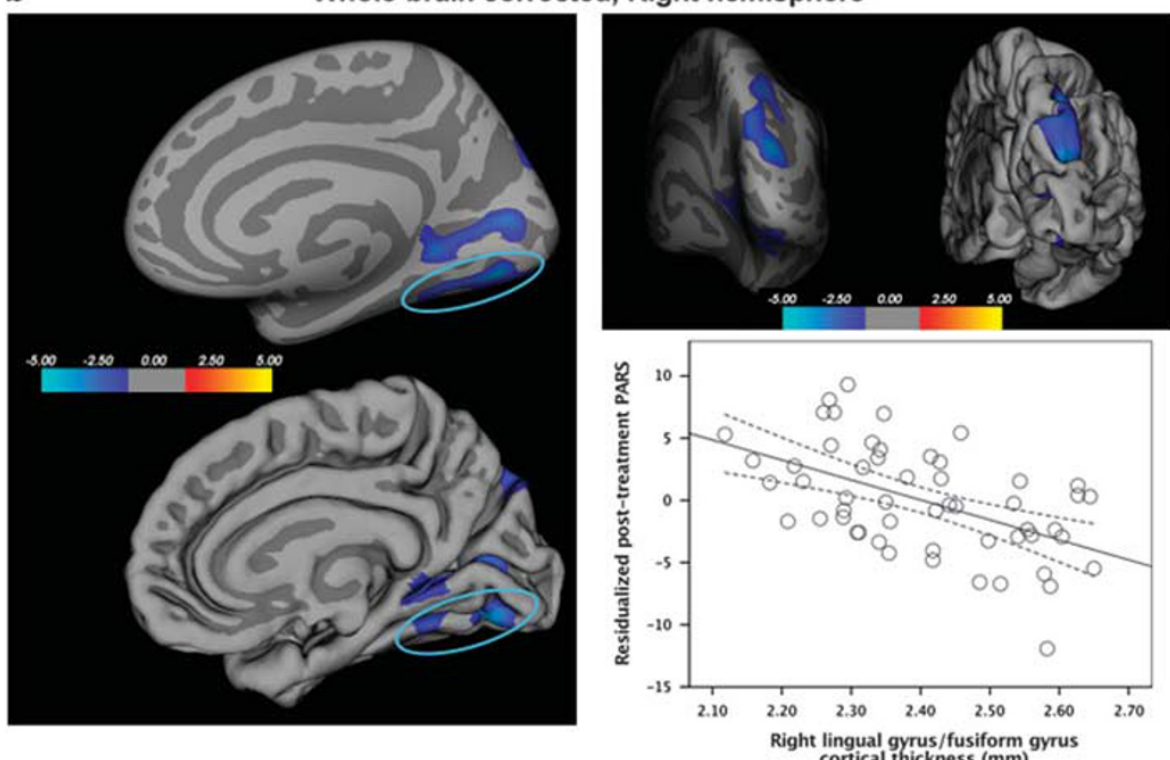

Figure 3 Associations of cortical thickness and anxiety treatment response. On the basis of the whole-brain vertex-wise correction ( $p<0.05$, corrected), thinner cortex in five clusters in the parietal and occipital cortices was associated with worse anxiety treatment response, ie, higher continuous anxiety symptom ratings post treatment controlling for baseline. Results are shown on both the inflated and pial surfaces. In the left hemisphere (a), one cluster was in superior parietal cortex, spanning the lateral occipital cortex and cuneus $(\beta=-0.49)$, and the other in inferior parietal cortex, extending to the supramarginal gyrus $(\beta=-0.55)$. The scatterplot shows average cortical thickness of one of the clusters (ie, superior parietal/lateral occipital cortex) on the $x$ axis and continuous treatment response (ie, post-treatment Pediatric Anxiety Rating Scale (PARS) scores, residualized for pre-treatment PARS scores) on the $y$ axis. In the right hemisphere (b), two clusters emerged in the lingual gyrus, one extending to the fusiform gyrus $(\beta=-0.52)$ and the other extending to the pericalcarine cortex $(\beta=-0.52)$, and another cluster in the superior parietal cortex, extending to the lateral occipital cortex ( $\beta=-0.49)$. The scatterplot shows average cortical thickness of one of the clusters (ie, lingual gyrus/fusiform gyrus) on the $x$ axis and continuous treatment response (ie, post-treatment PARS scores, residualized for pre-treatment PARS scores) on the $y$ axis.

and identify neurobiological factors that may give rise to altered thickness in the PFC.

In addition to group differences in vmPFC thickness, the current study also links baseline variation in anxiety with smaller hippocampal GMV, with small-to-medium effect sizes. This replicates prior findings of smaller hippocampal GMV in pediatric (Koolschijn et al, 2013; Mueller et al, 2013) and adult (Irle et al, 2010; Liao et al, 2011) anxiety. However, other studies find larger hippocampal GMV in adult anxiety (Machado-de-Sousa et al, 2014) or no differences in pediatric (De Bellis et al, 2000; Liao et al, 2014; Milham et al, 2005; Strawn et al, 2013) or adult anxiety (Brühl et al, 2014b; Frick et al, 2013; Talati et al, 2013). Aside from the hippocampus and vmPFC, the current study did not detect any differences 
in regions frequently targeted in anxiety neuroimaging research, ie, the amygdala, insula, and dorsal ACC. Indeed, these regions are rarely implicated in structural studies of pediatric anxiety (Supplementary Table S1) despite being the focus of considerable research. Importantly, both the pediatric and adult literatures are limited by small sample sizes, as most recruited fewer than 20 , and none more than 40, patients. Therefore, inconsistent findings may reflect low statistical power. To clarify these possibilities, even larger studies are needed.

In the current study, significant associations with anxiety diagnosis involved small-to-medium effect sizes (Cohen's $d=0.38-0.58)$. Structural imaging studies of anxiety disorders rarely yield large effect sizes. Morey et al (2009) estimated per group sample sizes needed to detect large effect sizes using FreeSurfer to be $n=14$ for the hippocampus and $n=23$ for the amygdala. With only $12-25$ patients per study, most structural neuroimaging studies of anxiety are underpowered. Thus, it remains unclear if large samples of more homogenous patient populations could find larger effect sizes. Strengths of the current study include medication-free patients, a relatively large sample, and a relatively homogenous phenotype. Despite these strengths, however, even more narrow phenotypes could be targeted in future work aimed to detect large effect sizes. This could include studies in more narrow age bands or in participants who are followed longitudinally and exhibit persistent anxiety across development. Alternatively, future research might combine structural MRI data with other parameters, such as fMRI, behavioral, and clinical measures, to create composite moderator variables with larger effect sizes needed to predict outcomes and inform treatments for anxiety. Indeed, clinical implications will emerge from findings integrated from a range of methodologies. For instance, functional MRI has larger effect sizes and is more tightly linked with psychological processes relative to structural MRI. However, structural MRI has better spatial resolution, and findings implicating structural perturbations in the hippocampus and vmPFC should increase interest in the functional perturbations of these regions.

In addition to anxiety phenotype, we also examined associations of neural structure with treatment response in a subset of 53 patients completing CBT. Controlling for baseline symptoms, higher clinician-rated anxiety symptoms (PARS) post treatment were related to thinner cortex in occipital and parietal cortices. Relatively few structural studies examine anxiety, and the even smaller number of imaging studies on anxiety treatment response predominantly examine adults using functional measures (Ball et al, 2014). Consistent with our occipital cortex findings, functional responses to emotional stimuli in higher-order visual cortex were associated with CBT response in adults with social anxiety disorder (Doehrmann et al, 2013). Similarly, visual cortex activations predicted long-term outcomes following exposure therapy in adults with specific phobia (Hauner et al, 2012). Of note, in the current study, parietal and occipital cortical thickness were related to CBT response but not baseline anxiety. Possible explanations for differential associations of brain measures with baseline anxiety and treatment response are that some treatment mechanisms might act by addressing underlying pathology, whereas others might act by engaging intact brain structures. While the current findings are preliminary and warrant replication in larger samples, they are most consistent with the latter explanation. Possibly, successful CBT might recruit intact circuitry, including parietal and occipital cortices, to regulate regions that are associated with aberrant function and/or structure in anxiety patients. While PFC thickness and hippocampal GMV related to anxiety at baseline, neither measure related to $\mathrm{CBT}$ response. Alternatively, nonsignificant treatment associations in regions related to baseline variation in anxiety, ie, vmPFC and hippocampus, may reflect false-negative findings, and greater statistical power associated with larger sample size is needed to detect such associations. Further research in larger samples is needed to assess structural perturbations in anxiety disorders, and to identify distinct and overlapping regions associated with baseline anxiety and treatment response in the same study.

Several important limitations of this study should be noted. First, despite being the largest neuroanatomical study of pediatric anxiety patients to date, the sample size is still relatively small for clinical phenotypes that fail to show large effect sizes. Future brain morphometry research is needed in much larger samples of psychiatrically ill and healthy youths, on the order of thousands rather than hundreds. For example, ongoing large-scale collaborative efforts have reported on brain structure in over 2000 patients with schizophrenia (Erp et al, 2016). While other large studies have acquired morphometry data in more than 1000 youths (Satterthwaite et al, 2014), these studies typically do not contain as many severely anxious, treatment-seeking patients as in the current report. Pediatric adaptations of such largescale clinical neuroscience efforts, via multi-site studies, mega-analyses, and meta-analyses, are needed to recruit larger samples of patients with features similar to those in the current study. This might clarify the degree to which pediatric anxiety disorders indeed are associated with small-to-medium effect sizes.

In addition to sample size, the broad age range is another limitation given that it spans puberty, as well as the continued development of brain structure and clinical features throughout adolescence. To recruit a sufficiently large sample of patients, however, participants ranged from 8 to 18 years old. Of note, there were no group differences in age, nor did our data indicate age-by-diagnosis interactions on brain structure. Although Ducharme et al (2014) demonstrated interactions of age and anxious/depressed symptoms on vmPFC thickness in over 300 healthy children and young adults, this study contained very few individuals with anxiety that was as severe as in the current study. The current study is the first to recruit over 50 treatment-seeking pediatric patients with anxiety and relate brain structure to treatment response. The large age range has the advantage of maximizing statistical power (ie, facilitating recruitment) when searching for preliminary evidence of phenotypic and treatment-related variability in morphometry. In post hoc analyses, age did not moderate the association of cortical thickness and treatment response in the parietal and occipital clusters (all $p s>0.054$ ), which remained significant when controlling for age (all ps $<0.004)$. Nonetheless, approaches are needed that test such age-related interactions in larger clinical samples of pediatric anxiety patients, ideally followed over time. Similarly, sex differences relate to both brain 
morphometry and the development of psychopathology. While there were no group differences in sex effects or sexby-diagnosis interactions on morphometry, the current sample may have been limited in statistical power. Future research might test for sex differences in much larger samples.

For the dimensional analyses, severity measures collected up to 2 months before or after scanning were used in order to maximize sample size, and anxious patients may have completed treatment sessions during this time. Nonetheless, continuous anxiety measures were unavailable in 43 participants (4 anxious, 39 healthy) due to research demands and subject burden. Consequently, reduced statistical power may have obscured associations with anxiety severity. Moreover, continuous analyses were based on parent and child report, rather than clinician-based ratings; the latter were acquired only in anxious patients due to feasibility and clinician burden. Higher effect sizes have been observed for clinician- relative to self-report symptom ratings (Cuijpers et al, 2010), and future neuroanatomical research might examine clinician-based continuous measures of anxiety in larger samples of both patients and HVs.

Another limitation involves possible inaccuracies of the FreeSurfer automated segmentation methods for generating subcortical volumes (Hanson et al, 2012; Morey et al, 2009; Wenger et al, 2014), which might contribute to either falsepositive or false-negative findings. However, we utilized this approach to compare the current findings with the broader literature, in which many large-scale studies utilized FreeSurfer methods (Frodl et al, 2017; Hoogman et al, 2017; Schmaal et al, 2016).

Additionally, our ability to draw firm conclusions regarding the specificity of our findings to anxiety disorders may be limited by the presence of comorbid disorders in the anxious group. However, we included patients with comorbidities to enhance generalizability, given that anxiety often co-occurs with other pediatric disorders.

Finally, secondary analyses examining treatment response were limited by the heterogeneity of ABMT delivered concurrently with $\mathrm{CBT}$ in three quarters of patients. Post hoc ABMT moderation analyses are reported in the Supplementary Information for thoroughness. Thus, heterogeneity limits the generalizability of our findings to traditional CBT without placebo/active ABMT, although only a third of patients received active ABMT.

In summary, the current study related structural brain alterations to pediatric anxiety disorders and treatment response in a relatively large sample of medication-free patients and HVs. Baseline variation in anxiety was associated with thicker vmPFC and smaller hippocampal GMV, with small-to-medium effect sizes. These findings are relevant to treatment-seeking populations of children and adolescents with anxiety. Future work might follow children longitudinally to identify structural perturbations associated with persistent anxiety across development. Finally, parietal and occipital cortical thickness were related to CBT response but not baseline anxiety, suggesting that some treatment mechanisms might act by engaging intact brain structures; however, these findings are preliminary and warrant replication in larger samples.

\section{FUNDING AND DISCLOSURE}

This research was supported by the Intramural Research Program of the National Institute of Mental Health/National Institutes of Health (NIMH/NIH), ZIAMH002781 (Pine), and based on Clinical Study Protocol 01-M-0192 and NCT\#: 00018057. The authors declare no conflict of interest.

\section{ACKNOWLEDGMENTS}

This study was presented as an abstract at the annual meeting of the Society of Biological Psychiatry, Toronto, Ontario, 2015.

\section{REFERENCES}

Adleman NE, Fromm SJ, Razdan V, Kayser RR, Dickstein DP, Brotman MA et al (2012). Cross-sectional and longitudinal abnormalities in brain structure in children with severe mood dysregulation or bipolar disorder. J Child Psychol Psychiatry 53: 1149-1156.

Ball TM, Stein MB, Paulus MP (2014). Toward the application of functional neuroimaging to individualized treatment for anxiety and depression. Depress Anxiety 31: 920-933.

De Bellis MD, Casey BJ, Dahl RE, Birmaher B, Williamson DE, Thomas KM et al (2000). A pilot study of amygdala volumes in pediatric generalized anxiety disorder. Biol Psychiatry 48: 51-57.

Birmaher B, Khetarpal S, Brent D, Cully M, Balach L, Kaufman J et al (1997). The Screen for Child Anxiety Related Emotional Disorders (SCARED): scale construction and psychometric characteristics. J Am Acad Child Adolesc Psychiatry 36: 545-553.

Britton JC, Grillon C, Lissek S, Norcross Ma, Szuhany KL, Chen G et al (2013). Response to learned threat: an fMRI study in adolescent and adult anxiety. Am J Psychiatry 170: 1195-1204.

Britton JC, Lissek S, Grillon C, Norcross MA, Pine DS (2011). Development of anxiety: the role of threat appraisal and fear learning. Depress Anxiety 28: 5-17.

Brühl AB, Delsignore A, Komossa K, Weidt S (2014a). Neuroimaging in social anxiety disorder-a meta-analytic review resulting in a new neurofunctional model. Neurosci Biobehav Rev 47: 260-280.

Brühl AB, Hänggi J, Baur V, Rufer M, Delsignore A, Weidt S et al (2014b). Increased cortical thickness in a frontoparietal network in social anxiety disorder. Hum Brain Mapp 35: 2966-2977.

Bryant RA, Felmingham K, Whitford TJ, Kemp A, Hughes G, Peduto A et al (2008). Rostral anterior cingulate volume predicts treatment response to cognitive-behavioural therapy for posttraumatic stress disorder. J Psychiatry Neurosci 33: 142-146.

Buhle JT, Silvers JA, Wager TD, Lopez R, Onyemekwu C, Kober H et al (2014). Cognitive reappraisal of emotion: a meta-analysis of human neuroimaging studies. Cereb Cortex 24: 2981-2990.

Cha J, Greenberg T, Carlson JM, Dedora DJ, Hajcak G, Mujica-Parodi LR (2014). Circuit-wide structural and functional measures predict ventromedial prefrontal cortex fear generalization: implications for generalized anxiety disorder. J Neurosci 34: 4043-4053.

Cuijpers P, Li J, Hofmann SG, Andersson G (2010). Self-reported versus clinician-rated symptoms of depression as outcome measures in psychotherapy research on depression: a metaanalysis. Clin Psychol Rev 30: 768-778.

Doehrmann O, Ghosh SS, Polli FE, Reynolds GO, Horn F, Keshavan A et al (2013). Predicting treatment response in social anxiety disorder from functional magnetic resonance imaging. JAMA Psychiatry 70: 87-97. 
Ducharme S, Albaugh MD, Hudziak JJ, Botteron KN, Nguyen T-V, Truong $\mathrm{C}$ et al (2014). Anxious/depressed symptoms are linked to right ventromedial prefrontal cortical thickness maturation in healthy children and young adults. Cereb Cortex 24: 2941-2950.

Erp TGM, van, Hibar DP, Rasmussen JM, Glahn DC, Pearlson GD, Andreassen OA et al (2016). Subcortical brain volume abnormalities in 2028 individuals with schizophrenia and 2540 healthy controls via the ENIGMA consortium. Mol Psychiatry 21: 547-553.

Etkin A, Wager TD (2007). Functional neuroimaging of anxiety: a meta-analysis of emotional processing in PTSD, social anxiety disorder, and specific phobia. Am J Psychiatry 164: 1476-1488.

Frick A, Howner K, Fischer H, Eskildsen SF, Kristiansson M, Furmark T (2013). Cortical thickness alterations in social anxiety disorder. Neurosci Lett 536: 52-55.

Frodl T, Janowitz D, Schmaal L, Tozzi L, Dobrowolny H, Stein DJ et al (2017). Childhood adversity impacts on brain subcortical structures relevant to depression. J Psychiatr Res 86: 58-65.

Fullana MA, Cardoner N, Alonso P, Subirà M, López-Solà C, Pujol J et al (2014). Brain regions related to fear extinction in obsessivecompulsive disorder and its relation to exposure therapy outcome: a morphometric study. Psychol Med 44: 845-856.

Gold AL, Brotman MA, Adleman NE, Lever SN, Steuber ER, Fromm SJ et al (2016). Comparing brain morphometry across multiple childhood psychiatric disorders. J Am Acad Child Adolesc Psychiatry 55: 1027-1037.

Hanson JL, Suh JW, Nacewicz BM, Sutterer MJ, Cayo AA, Stodola DE et al (2012). Robust automated amygdala segmentation via multi-atlas diffeomorphic registration. Front Neurosci 6: 166.

Hauner KK, Mineka S, Voss JL, Paller KA (2012). Exposure therapy triggers lasting reorganization of neural fear processing. Proc Natl Acad Sci USA 109: 9203-9208.

Hoexter MQ, Souza Duran FL, de, D'Alcante CC, Dougherty DD, Shavitt RG, Lopes AC et al (2012). Gray matter volumes in obsessive-compulsive disorder before and after fluoxetine or cognitive-behavior therapy: a randomized clinical trial. Neuropsychopharmacology 37: 734-745.

Hoogman M, Bralten J, Hibar DP, Mennes M, Zwiers MP, Schweren LSJ et al (2017). Subcortical brain volume differences in participants with attention deficit hyperactivity disorder in children and adults: a cross-sectional mega-analysis. Lancet Psychiatry 4: 310-319.

Hutton C, Draganski B, Ashburner J, Weiskopf N (2009). A comparison between voxel-based cortical thickness and voxelbased morphometry in normal aging. Neuroimage 48: 371-380.

Irle E, Ruhleder M, Lange C, Seidler-Brandler U, Salzer S, Dechent P et al (2010). Reduced amygdalar and hippocampal size in adults with generalized social phobia. J Psychiatry Neurosci 35: 126-131.

Jones JE, Jackson DC, Chambers KL, Dabbs K, Hsu DA, Stafstrom CE et al (2015). Children with epilepsy and anxiety: Subcortical and cortical differences. Epilepsia 56: 283-290.

Kaufman J, Birmaher B, Brent D, Rao U, Flynn C, Moreci P et al (1997). Schedule for Affective Disorders and Schizophrenia for School-Age Children-Present and Lifetime Version (K-SADSPL): initial reliability and validity data. J Am Acad Child Adolesc Psychiatry 36: 980-988.

Kendall P, Hedtke K (2006). The Coping Cat Program Workbook. Workbook Publishing: Ardmore, PA.

Koolschijn PCMP, van IJzendoorn MH, Bakermans-Kranenburg MJ, Crone EA (2013). Hippocampal volume and internalizing behavior problems in adolescence. Eur Neuropsychopharmacol 23: 622-628.

Kujawa A, Swain JE, Hanna GL, Koschmann E, Simpson D, Connolly $S$ et al (2016). Prefrontal reactivity to social signals of threat as a predictor of treatment response in anxious youth. Neuropsychopharmacology 41: 1983-1990.
Liao M, Yang F, Zhang Y, He Z, Su L, Li L (2014). Lack of gender effects on gray matter volumes in adolescent generalized anxiety disorder. J Affect Disord 155: 278-282.

Liao W, Xu Q, Mantini D, Ding J, MacHado-De-Sousa JP, Hallak JEC et al (2011). Altered gray matter morphometry and resting-state functional and structural connectivity in social anxiety disorder. Brain Res 1388: 167-177.

Machado-de-Sousa JP, Osório F de L, Jackowski AP, Bressan RA, Chagas MHN, Torro-Alves N et al (2014). Increased amygdalar and hippocampal volumes in young adults with social anxiety. PLoS ONE 9: e88523.

McClure EB, Adler A, Monk CS, Cameron J, Smith S, Nelson EE et al (2007). fMRI predictors of treatment outcome in pediatric anxiety disorders. Psychopharmacology 191: 97-105.

Milad MR, Quinn BT, Pitman RK, Orr SP, Fischl B, Rauch SL (2005). Thickness of ventromedial prefrontal cortex in humans is correlated with extinction memory. Proc Natl Acad Sci USA 102: 10706-10711.

Milad MR, Quirk GJ (2012). Fear extinction as a model for translational neuroscience: ten years of progress. Annu Rev Psychol 63: 129-151.

Milham MP, Nugent AC, Drevets WC, Dickstein DP, Leibenluft E, Ernst $M$ et al (2005). Selective reduction in amygdala volume in pediatric anxiety disorders: a voxel-based morphometry investigation. Biol Psychiatry 57: 961-966.

Mohatt J, Bennett SM, Walkup JT (2014). Treatment of separation, generalized, and social anxiety disorders in youths. $A m \mathrm{~J}$ Psychiatry 171: 741-748.

Morey RA, Petty CM, Xu Y, Hayes JP, Wagner HR, Lewis DV et al (2009). A comparison of automated segmentation and manual tracing for quantifying hippocampal and amygdala volumes. Neuroimage 45: 855-866.

Mueller SC, Aouidad A, Gorodetsky E, Goldman D, Pine DS, Ernst M (2013). Gray matter volume in adolescent anxiety: an impact of the brain-derived neurotrophic factor $\mathrm{Val}(66)$ Met polymorphism? J Am Acad Child Adolesc Psychiatry 52: 184-195.

Murray EA, Wise SP, Graham KS (2017). The Evolution of Memory Systems. Oxford University Press: Oxford, UK.

Panizzon MS, Fennema-Notestine C, Eyler LT, Jernigan TL, Prom-Wormley E, Neale $M$ et al (2009). Distinct genetic influences on cortical surface area and cortical thickness. Cereb Cortex 19: 2728-2735.

Price JL, Drevets WC (2010). Neurocircuitry of mood disorders. Neuropsychopharmacology 35: 192-216.

Raznahan A, Shaw P, Lalonde F, Stockman M, Wallace GL, Greenstein D et al (2011). How does your cortex grow? J Neurosci 31: 7174-7177.

The Research Units on Pediatric Psychopharmacology Anxiety Study Group (2001). Fluvoxamine for the treatment of anxiety disorders in children and adolescents. N Engl J Med 344: 1279-1285.

The Research Units on Pediatric Psychopharmacology Anxiety Study Group (2002). The Pediatric Anxiety Rating Scale (PARS): development and psychometric properties. J Am Acad Child Adolesc Psychiatry 41: 1061-1069.

Satterthwaite TD, Elliott MA, Ruparel K, Loughead J, Prabhakaran K, Calkins ME et al (2014). Neuroimaging of the Philadelphia neurodevelopmental cohort. Neuroimage 86: 544-553.

Schmaal L, Veltman DJ, Erp TGM, van, Sämann PG, Frodl T, Jahanshad $\mathrm{N}$ et al (2016). Subcortical brain alterations in major depressive disorder: findings from the ENIGMA Major Depressive Disorder working group. Mol Psychiatry 21: 806-812.

Shang J, Fu Y, Ren Z, Zhang T, Du M, Gong Q et al (2014). The common traits of the ACC and PFC in anxiety disorders in the DSM-5: meta-analysis of voxel-based morphometry studies. PLoS ONE 9: e93432.

Strawn JR, Hamm L, Fitzgerald DA, Fitzgerald KD, Monk CS, Phan KL (2015). Neurostructural abnormalities in pediatric anxiety disorders. J Anxiety Disord 32: 81-88. 
Strawn JR, John Wegman C, Dominick KC, Swartz MS, Wehry AM, Patino LR et al (2014). Cortical surface anatomy in pediatric patients with generalized anxiety disorder. J Anxiety Disord 28: 717-723.

Strawn JR, Wehry AM, Chu W-J, Adler CM, Eliassen JC, Cerullo MA et al (2013). Neuroanatomic abnormalities in adolescents with generalized anxiety disorder: a voxel-based morphometry study. Depress Anxiety 30: 842-848.

Syal S, Hattingh CJ, Fouché J-P, Spottiswoode B, Carey PD, Lochner C et al (2012). Grey matter abnormalities in social anxiety disorder: a pilot study. Metab Brain Dis 27: 299-309.

Talati A, Pantazatos SP, Schneier FR, Weissman MM, Hirsch J (2013). Gray matter abnormalities in social anxiety disorder: primary, replication, and specificity studies. Biol Psychiatry 73: $75-84$.
Wenger E, Mårtensson J, Noack H, Bodammer NC, Kühn S, Schaefer S et al (2014). Comparing manual and automatic segmentation of hippocampal volumes: reliability and validity issues in younger and older brains. Hum Brain Mapp 35: 4236-4248.

White LK, Sequeira S, Britton JC, Brotman MA, Gold AL, Berman E et al (in press). Complementary features of attention bias modification therapy and cognitive behavioral therapy in pediatric anxiety disorders. Am J Psychiatry.

Wierenga LM, Langen M, Oranje B, Durston S (2014). Unique developmental trajectories of cortical thickness and surface area. Neuroimage 87: 120-126.

Winkler AM, Kochunov P, Blangero J, Almasy L, Zilles K, Fox PT et al (2010). Cortical thickness or grey matter volume? The importance of selecting the phenotype for imaging genetics studies. Neuroimage 53: 1135-1146.

Supplementary Information accompanies the paper on the Neuropsychopharmacology website (http://www.nature.com/npp) 